\title{
SATELLITE AND AERIAL IMAGE DECONVOLUTION USING AN EM METHOD WITH COMPLEX WAVELETS ${ }^{\star}$
}

\author{
André Jalobeanu ${ }^{1}$, Robert D. Nowak ${ }^{2}$, Josiane Zerubia ${ }^{1}$, Mario A. T. Figueiredo ${ }^{3}$ \\ 1 Ariana, joint research group CNRS/INRIA/UNSA, INRIA Sophia Antipolis, France - firstname.lastname@inria.fr \\ 2 Dept. of Electrical and Computer Eng., Rice University, Houston, TX 77005, USA - nowak@rice. edu \\ 3 Institute of Telecommunications, Instituto Superior Técnico, 1049-001 Lisboa, Portugal - mtf@lx.it.pt \\ * This work was done while the second author was visiting professor at Ariana, partially supported by an INRIA fellowship.
}

\begin{abstract}
In this paper, we present a new deconvolution method, able to deal with noninvertible blurring functions. To avoid noise amplification, a prior model of the image to be reconstructed is used within a Bayesian framework. We use a spatially adaptive prior, defined with a complex wavelet transform in order to preserve shift invariance and to better restore variously oriented features. The unknown image is estimated by an EM technique, whose E step is a Landweber update iteration, and the $M$ step consists of denoising the image, which is achieved by wavelet coefficient thresholding. The new algorithm has been applied to high resolution satellite and aerial data, showing better performance than existing techniques when the blurring process is not invertible, like motion blur for instance.
\end{abstract}

\section{INTRODUCTION}

The problem considered in this paper is the reconstruction of high resolution satellite or aerial images from blurred and noisy data. Such images are corrupted by different sources of blur: the optical system, the integration over the pixels, and the motion. The motion blur is generally difficult to handle, because it is not invertible, since the transfer function has zeros (corresponding to the Fourier transform of a box function, for instance). Inverting the observation process without amplifying the noise in this case is difficult.

In this paper, we propose a solution based on two original works proposed in [5, 9]. The former uses a spatially adaptive prior model of the image based on complex wavelets, while the latter enables us to invert any type of blur just by using a denoising algorithm.

The observation model is represented by

$$
Y=h \star X+N \quad \text { with } N \sim \mathcal{N}_{N}\left(0, \sigma^{2} I\right),
$$

where $Y$ is the observed data, $X$ is the original image and $\star$ denotes a circular convolution. The Point Spread Function (PSF) $h$ is supposed to be known. $N$ is the additive noise and is supposed to be Gaussian, white and stationary, of known variance $\sigma^{2}$ ( $I$ denotes the identity matrix).

Herein, $\mathcal{N}_{W}(0, \Sigma)$ denotes the multivariate Gaussian density function for a vector $W$, with zero mean and covariance matrix $\Sigma$.

The noise $N$ is decomposed in the following way:

$$
Y=h \star\left(X+N^{0}\right)+N^{1} \text { with }\left\{\begin{array}{l}
N^{0} \sim \mathcal{N}_{N^{0}}\left(0, \Sigma^{0}\right) \\
N^{1} \sim \mathcal{N}_{N^{1}}\left(0, \Sigma^{1}\right)
\end{array}\right.
$$

such as $N^{0}$ and $N^{1}$ are independent Gaussian processes with respective covariance matrices $\Sigma^{0}$ and $\Sigma^{1}$. This decomposition was initially proposed by in [9], with both $N^{0}$ and $N^{1}$ white noises. A similar approach has been used in [10] in the case of Poisson noise.

We choose to define the covariance matrices in such a way to recover the observation equation (1), such that $N=$ $h \star N^{0}+N^{1}$. Thus, the noise remains white. We choose $N^{0}$ as a white noise, to keep the main interest of the algorithm proposed in [9], consisting of replacing the deconvolution by a denoising technique. Let us also suppose that these matrices are diagonalized by a Fourier transform (like the convolution operator) to simplify the problem. Then, the white noise condition is expressed as

$$
\sigma^{2}=\mathcal{F}[h]_{u, v}^{2}\left(\sigma_{u, v}^{0}\right)^{2}+\left(\sigma_{u, v}^{1}\right)^{2},
$$

where $\mathcal{F}[$.$] denotes the Fourier transform and \sigma_{u, v}^{0}, \sigma_{u, v}^{1}$ the eigenvalues of $\Sigma^{0}$ and $\Sigma^{1}$. Let $a$ be a positive real number, and $H$ the blurring operator related to the convolution by $h$. The condition (3) is fulfilled if we have

$$
\Sigma^{0}=a \sigma^{2} I \quad \text { and } \quad \Sigma^{1}=\sigma^{2}\left(I-a H^{t} H\right) .
$$

\section{EM ALGORITHM}

To estimate the unknown image $X$, we choose a Bayesian approach, consisting of computing the MAP

$$
\hat{X}=\arg \max _{X} P(X \mid Y) .
$$


The posterior density $P(X \mid Y)$ can be written as the product of the observation density $P(Y \mid X)$ by the prior density $P(X)$, using Bayes' rule. The former is given by the observation model (1), while the latter is chosen in order to model the properties of the unknown image. In this paper, we define a model expressed in the wavelet domain, but other models can be used without loss of generality.

An efficient way to perform the MAP computation is to use the EM algorithm [3]. This method is designed to solve problems when some data are missing. The main interest of applying EM is to get an optimization problem which is simpler than the original one, thanks to the missing data introduction at each iteration of the algorithm.

The user can freely choose the missing data and the parameters to be estimated when using an EM-like procedure. In the case presented herein, it is useful to consider the noise decomposition (2) to define the missing data: $z=X+N^{0}$ [9]. The observation model remains globally unchanged, but we consider two steps instead of one: first add $N^{0}$ to $X$ to get $z$, and then $Y=h \star z+N^{1}$ (see. Fig. 1).

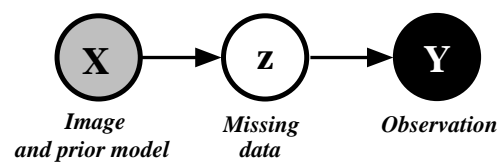

Fig. 1. Dependence graph of the model used in the proposed method, representing the density $P(X, Y, z)$.

The expression of the complete model, including the missing data, is then given by

$$
\begin{aligned}
& P(X, Y, z)=P(Y \mid z) P(z \mid X) P(X) \\
& \text { with }\left\{\begin{array}{l}
P(z \mid X)=\mathcal{N}_{z}\left(X, \Sigma^{0}\right) \\
P(Y \mid z)=\mathcal{N}_{Y}\left(h \star z, \Sigma^{1}\right)
\end{array}\right.
\end{aligned}
$$

The E step at iteration $n$ consists of computing the expectation of $\log P(Y, z \mid X) P(X)$ with $z \sim P\left(z \mid Y, X^{n}\right)$, denoted $\bar{z}$. After calculus in the Fourier space, we obtain

$$
\bar{z}=\mathbb{E}_{z \mid Y, X^{n}}[z]=X^{n}+a h^{*} \star\left(Y-h \star X^{n}\right),
$$

which looks like the Landweber iteration [8], with an acceleration factor $a$, which has to be constrained within the interval $(0,2)$ for stability reasons.

The $\mathrm{M}$ step is the following:

$$
X^{n+1}=\arg \max _{X \in \Omega_{S}}\left[\frac{\|X-\bar{z}\|^{2}}{2 a \sigma^{2}}+\log P(X)\right] .
$$

This equation corresponds to computing the MAP using an observed image $\bar{z}$, noisy but not blurred. The M step is therefore a denoising step, assuming a white noise of variance $a \sigma^{2}$ and a prior model $P(X)$. The corresponding iterative algorithm is illustrated by Fig. 2 .

\section{COMPLEX WAVELETS DENOISING}

\subsection{The prior model}

We propose to model the image using complex wavelets. Satellite and aerial images exhibit both scale invariance and non stationarity properties, which can be captured at the same time by a wavelet transform. However, real dyadic wavelet transforms have two drawbacks when they are applied to image denoising.

First, they are not shift invariant. This means that denoising the image by thresholding the coefficients often produces artefacts, depending on the alignment between the image features and the wavelet basis. Second, they are not rotation invariant, since artefacts can also appear near round features. Indeed, such transforms essentially act like multiscale derivation operators w.r.t. rows and columns, which is a drawback for diagonal edges for instance.

An elegant manner to ensure approximate translational and rotational invariances is to use complex wavelets [7]. The Complex Wavelet Transform (CWT) is obtained by using 4 parallel interleaved trees, corresponding to real separable biorthogonal wavelet transforms which are shifted and subsampled differently to optimize the shift invariance. The coefficients of the real transforms are combined together to form complex coefficients, enabling us to separate 6 orientations. The resulting directional selectivity enables us to capture the small oriented features, the diagonal edges and the textures present in satellite or aerial images, thus providing an efficient separation between signal and noise in the wavelet domain.

Then, we model the unknown image by a non stationary multiscale Gaussian model. If we denote the coefficients of the CWT of $X$ by $\xi_{t}$, we have

$$
P\left(\xi_{t}\right)=\mathcal{N}_{\xi}\left(0, s_{t}^{2} I\right),
$$

where $s_{t}^{2}$ is the local variance of the real or imaginary parts of $\xi_{t}$, which are supposed to be independent.

\subsection{Adaptive parameter estimation and denoising}

The observation model expressed in the wavelet domain is simply $\zeta_{t}=\xi_{t}+\tilde{N}^{0}$ where $\zeta$ is the CWT of $z$ and $N^{0}$ represents a bidimensional white Gaussian noise of variance $2 a \sigma^{2}$, the real and imaginary parts being independent. If the parameters $s_{t}$ are known, the MAP is given by

$$
\hat{\xi}_{t}=\frac{s_{t}^{2}}{s_{t}^{2}+a \sigma^{2}} \zeta_{t}
$$

This is an adaptive thresholding. Since it does not modify the phase, the shift invariance property is preserved.

To estimate the adaptive parameters, we use a hybrid approach derived from the algorithm COWPATH 2 [5]. It 
consists of performing the estimation from a good approximation of the original image, within a complete data framework, which enables us to get a simple and robust estimate. To compute the needed approximate original image, we use an edge-preserving deconvolution method called RHEA [6], which consists of performing a non quadratic regularization using automatically estimated parameters. The CWT of the result is filtered using a non informative Jeffrey's prior to remove the residual noise, which gives us the wavelet coefficients denoted $\tilde{\xi}_{t}$, i.e. the complete data.

Then, the parameter estimation step from $\tilde{\xi}_{t}$ is simply $\hat{s}_{t}^{2}=\left|\tilde{\xi}_{t}\right|^{2} / 2$. Finally, the M step becomes

$$
\hat{\xi}_{t}=\frac{\tilde{\xi}_{t}^{2}}{\tilde{\xi}_{t}^{2}+2 a \sigma^{2}} \zeta_{t} .
$$

\section{THE PROPOSED ALGORITHM: EM-DEC}

The EM-DEC ("E M DEConvolution") algorithm, based on an EM method and a spatially adaptive complex wavelet prior, is the following (see Fig. 2):

- Initialization: $X^{0}=Y, a \in(0,2)$

- Compute $\tilde{X}$ from $Y$ using RHEA [6]

- Direct CWT of $\tilde{X}$ and noise thresholding to get $\tilde{\xi}$

- Repeat until convergence:

- E step: Landweber iteration, Eqn. (8)

- M step: complex wavelet denoising

- Direct CWT

- Denoising using Eqn. (12)

- Inverse CWT to get $X^{n+1}$.

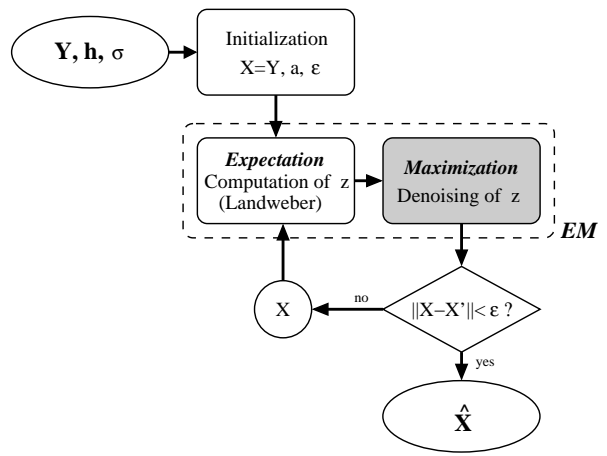

Fig. 2. Diagram of the EM-DEC algorithm.

This method enables us to deal with any deconvolution problem using only a denoising algorithm at each step. It also allows to use sophisticated wavelet-based models, which could be difficult to handle within a deconvolution context. But due to the denoising step, the computation is quite easy, which is usually not the case for methods based on gradient descent, like in $[2,11]$ for instance.

Furthermore, the approach presented here does not require the blurring operator to be invertible like in wavelet packet or wavelet-vaguelette techniques such as [4]. To illustrate this point, we have chosen to make experiments with a $3 \times 3$ box convolution kernel, whose transfer function has zeros in the frequency space.

The necessary conditions of convergence of EM-DEC are the same as for the EM method: the function in Equ. (9) has to be strictly concave to ensure the convergence to the global optimum, independently on the initialization. The chosen Gaussian prior fulfills this condition. It is not true for other priors (such as Generalized Gaussian for instance), which can lead to local optima depending on the initialization.

Another necessary condition is that the acceleration parameter $a$ must be strictly lower than 2, like in the Landweber method. A value close to this bound gives the highest convergence rate. However, this rate can be strongly enhanced by choosing a better initialization than the observed image $Y$, as illustrated by Fig. 3, related to the experiment shown in next section.

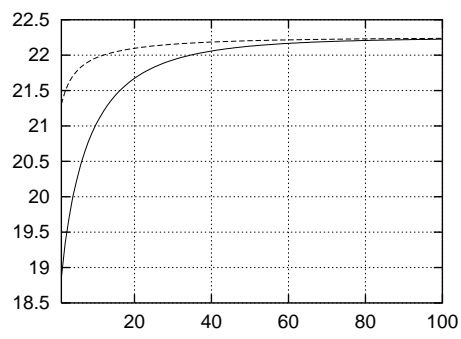

Fig. 3. SNR (in $\mathrm{dB}$ ) as a function of the number of iterations, for the algorithm EM-DEC. Solid: initalization using the observed image, dashed: initalization using Wiener filter.

The idea of using a denoising method at each step of an iterative deconvolution method has already been proposed in [1]. The authors propose to denoise the residual $Y-h \star$ $X^{n}$ at each step, but there is neither proof of convergence, nor prior model of the unknown solution.

\section{RESULTS}

Fig. 4 shows a $128 \times 128$ area extracted from an image provided by the French Space Agency (CNES), this is the original image $X$. The blurring kernel is a $3 \times 3$ box function. We have $\sigma^{2}=2$.

The proposed algorithm has been compared to other algorithms: Wiener filter, which gives poor results (noisy image and blurred edges); RHEA method [6], which gives sharp edges (but still noisy homogeneous areas); COWPATH 2 [5], which does not work properly because of the non invertible blurring function; and EM-DEC 0, which is a simplified version of the proposed method, where the denoising step is a simple soft thresholding (with an optimal threshold). The results and the related SNRs are shown in Fig. 4. 
The new method performs better than other ones, and provides sharp edges, clear constant areas, and sharp oriented features and textures, thanks to complex wavelets. Each iteration needs 2 FFTs and 2 CWTs, of complexity $\mathrm{O}(n \log n)$ where $n$ is the number of pixels.

\section{CONCLUSION}

We have proposed a new deconvolution algorithm based on the EM procedure, which enables us to solve deconvolution problems using only a denoising technique. This approach consists of alternating a Landweber step and a denoising step. The latter is performed using a complex wavelet transform, ensuring both translational and rotational invariance properties, and a spatially adaptive prior model. The algorithm can deal with any type of blur, and outperforms state of the art wavelet packet deconvolution techniques when the blur is not invertible.

\section{REFERENCES}

[1] A. Bijaoui, J-L. Starck, and F. Murtagh. Multiresolution support applied to image filtering and restoration. Comp. Vision, Graphics and Image Proc. A., 1995.

[2] P. de Rivaz and N. Kingsbury. Bayesian image deconvolution and denoising using complex wavelets. IEEE Proc. of ICIP, Thessaloniki, Greece, Oct. 2001.

[3] A. Dempster, N. Laird, and D. Rubin. Maximum Likelihood from incomplete data via the EM algorithm. Journal of Roy. Stat. Soc. B, 39:1-38, 1977.

[4] D. Donoho. Nonlinear solution of linear inverse problems by wavelet-vaguelette decomposition. App. and Comp. Harmonic Analysis, 2:101-126, 1995.

[5] A. Jalobeanu, L. Blanc-Féraud, and J. Zerubia. Satellite image deconvolution using complex wavelet packets. In Proc. of ICIP, Vancouver, Sept. 2000.

[6] A. Jalobeanu, L. Blanc-Féraud, and J. Zerubia. Hyperparameter estimation for satellite image restoration using a MCMC Maximum Likelihood method. Pattern Recognition, 35(2):341-352, 2002.

[7] N. Kingsbury. The dual-tree complex wavelet transform: a new efficient tool for image restoration and enhancement. In Proc. of EUSIPCO, pages 319-322, Rhodes, Greece, 1998.

[8] L. Landweber. An iteration formula for Fredholm integral equations of the 1rst kind. Amer. J. Math., 73:615-624, 1951.

[9] R.D. Nowak and M.A.T. Figueiredo. Fast wavelet-based image deconvolution using the EM algorithm. Proc. of the Asilomar Conf. on Signals, Systems and Computers, Pacific Grove, CA, Nov. 2001.

[10] R.D. Nowak and E.D. Kolaczyk. A statistical multiscale framework for Poisson inverse problems. IEEE Trans. on IT, 46(5):1811-1825, 2000.

[11] Y. Wan and R.D. Nowak. A wavelet-based statistical model for image restoration. IEEE Proc. of ICIP, Thessaloniki, Greece, Oct. 2001.

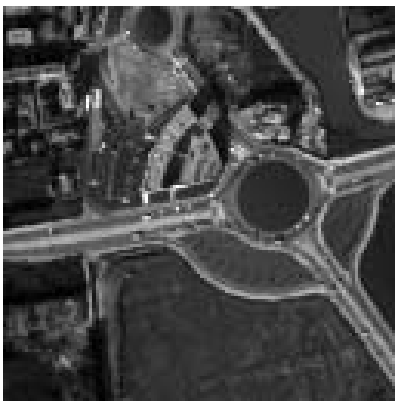

Original image

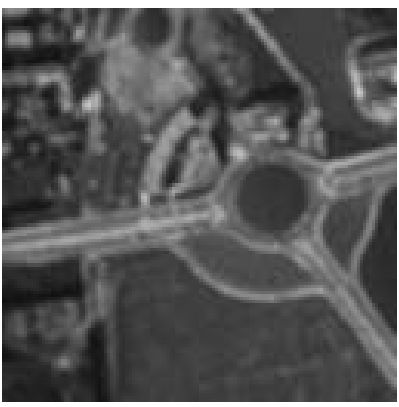

Observed image, $17.0 \mathrm{~dB}$

RHEA, $21.8 \mathrm{~dB}$

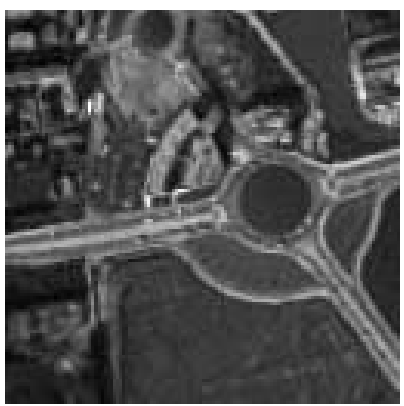

EM-DEC 0, $21.8 \mathrm{~dB}$

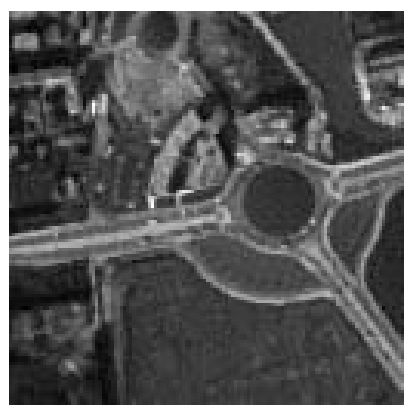

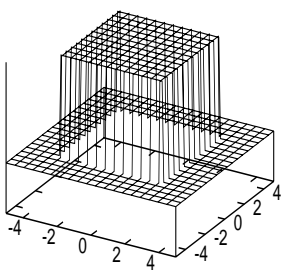

Blurring kernel ( $3 \times 3$ box $)$

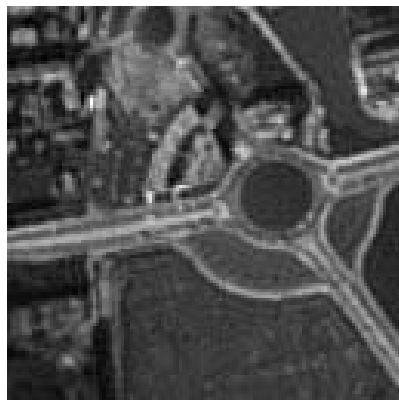

Wiener, $20.7 \mathrm{~dB}$

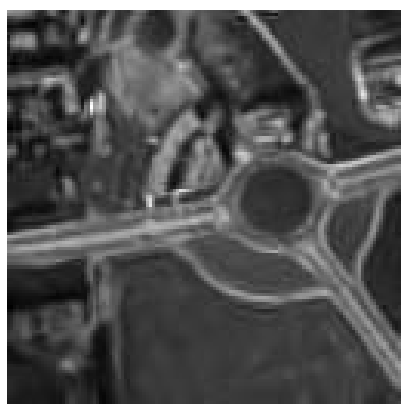

COWPATH 2, $19.0 \mathrm{~dB}$

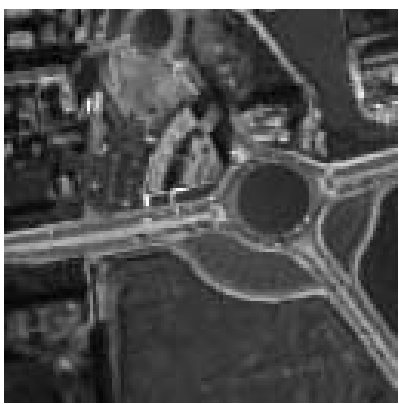

EM-DEC, $22.2 \mathrm{~dB}$
Fig. 4. Original, observed and restored image with different methods. The area is extracted from an aerial image of Nîmes (C) CNES. 